\title{
Self-Sufficient Energy Harvesting in Robots using Nanotechnology
}

\section{Basma El Zein*}

Dar Al Hekma University, Saudi Arabia

\begin{abstract}
Latest research and technology development have been focusing on improving the performance and the efficiency of intelligent and automated machines / systems used in human services, factories, transportation means, space exploration and may others applications. As the tech world is shrinking rapidly, industries are seeking to miniaturize the devices to the level of nano-machines /nanorobots without compromising their efficiency. Scientists have made significant progress in nanoroboics research field but have not officially released their new products. On the other hand, they opened a new world of discoveries, possibilities and applications in different fields such as military defense, medicine, industry, space exploration, energy. In this article, we will present the role of nanotechnology and nanostructures in energy harvesting systems used in robots to be able to sense and adapt heat, light, sounds, surface texture and chemicals from the environment, as well as to move, communicate and perform complex calculations.
\end{abstract}

Keywords: Nano robots; Automated machines; Pneumatic sensors

\section{Introduction}

Having revolutionary impact on industrial production route, nanotechnology, the engineering of functional system at the atomic scale or nano-scale $\left(10^{-9} \mathrm{~m}\right)=10 \AA$, is expected to bring many benefits in the automotive industry and robotics. The advances in nanotechnology are very promising to improve our quality of life in all fields ranging from healthcare to electronics. Nanostructures and nano material will be extensively studied and then employed in the design of nano-devices to reduce the material and fabrication cost and improves the efficiency. Being an electromechanical device, a robot is capable of interacting with its environment and is controlled and commanded by a computer program and electronic circuits. Generally a robot is composed of a movable body, actuator with an electric motor, hydraulic or pneumatic sensors to communicate with the programmable brain, through electric and electronic circuits. Nanorobotics, one of the merging fields of robotics at the nanoscale, has a major objective of shrinking device size to nano scale dimensions. A Nanorobot, the controllable machine at the nanometer, is composed of nano-scale component with a smart structure that can actuate, sense, signal, and process information, all at Nano scale. Having so many features such as durability, efficiency, effectiveness and low cost, nano-robots, find wide applications in the field of medicine and space technology. Nano robotic systems deal with vast variety of sciences, ranging from quantum molecular dynamics, to kinematic analysis. Therefore, It depends on the type of nano material and nanostructure that have been employed.

\section{Self Sufficient Energy Harvesting In Nano Robots}

As human intervention is not needed, it is important to use the ambient energy (present in the environment) to convert it to electrical energy to drive small electrical and electronic systems making them self-sufficient. For example : One of the applications of nano-robots in the medical field is its inside of the human body to cure many diseases such as diabetes, tumors, blood clots and many others, with no harm to the body. These nano robots are very sensitive to human body temperature, acoustic signals, blood flow, body movement, respiration, body heat...; therefore they can get the energy from the body itself. Power can be supplied when the source is available (battery less), but the most important is to supply electricity when it is needed to match the demand with the supply. Our environment contains a variety of sources available for harvesting presented in different forms not limited to mechanical, thermal and light or solar (Figure 1).

\section{Nanotechnology Role}

New innovative and improved products are expect5ed to enter the market due to the promising benefits of Nanotechnology. This later has showed many benefits in different applications in aerospace, agriculture, construction, cosmetics, defense electronics, environment, food, textile and energy. Due to the progress of Nanotechnology, nanostructures, semiconductors materials have facilitated the cost reduction and improvement in energy conversion efficiency. In this section we will focus on the use of nano wire in energy harvesting to be employed in nano-robots. Nano-wires or one dimensional nanostructure have unique optical and electrical properties which makes them more applicable in energy harvesting devices. These elongated solid nanostructure, have high aspect ratio, and offer great possibilities for further development of many optoelectronic devices and sensors, with numerous possibilities for studying exciting physical

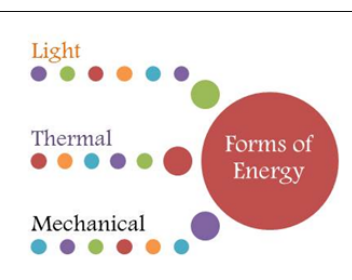

Figure 1: different forms of energy.

*Corresponding author: Basma El Zein, Dar Al Hekma University, Saudi Arabia, Tel: 9665046643 49; E-mail: basma@ieee.org

Received October 07, 2013; Accepted December 04, 2013; Published December 07, 2013

Citation: El-Zein B (2013) Self-Sufficient Energy Harvesting in Robots using Nanotechnology. Adv Robot Autom 2: 113. doi: 10.4172/2168-9695.1000113

Copyright: ( $2013 \mathrm{El}$-Zein B. This is an open-access article distributed under the terms of the Creative Commons Attribution License, which permits unrestricted use, distribution, and reproduction in any medium, provided the original author and source are credited. 
phenomenon arising from carrier confinement to large surface to volume ratio [1].

\section{Nanostructures in energy harvesting}

New nano material's and nanostructures are expected to make a difference in reducing $\mathrm{CO}_{2}$ generation, using renewable/clean energy, reducing manufacturing cost, improving performance and efficiency of energy harvesting devices such as light emitting diodes(LED), batteries, solar cells, fuel cells and many others . Recently, an increased motivation was noticed that employ nanostructures in energy harvesting and energy storage devices. These include: (a) bulk nano structured materials [3D]; (b) quantum wells [2D]; (c) nano wires [1D]; and (d) quantum dots/nano particles [0D]. Nano wires (NW), elongated solid nanostructure, are semiconducting or metallic structures having a high aspect ratio. Nano wires have been extensively studied in the last decade; and will be presented in the next paragraphs with their application in different forms of energy harvesting.

\section{Light and solar energy harvesting}

Recently, solar cell and nanowire researches are considered as hot topics within science and engineering [2]. The need for higher solar cell efficiencies at lower cost has become crucial, and the control of nanostructures has affected the performance of electronic and optical devices [3]. The unique geometry of Nanowires array provides low optical reflection and enhances light trapping and absorption (Figure 2 ). The free standing NWs array allows the incident light to scatter within its open interiors, which is different from the easy reflection of incident light on the surface of nano-crystallines film. The scattering improves the efficiency of light absorption by increasing the photon path length and diminishing the reflection of incident light. The photogenerated electron -hole pairs in bulk thin film tend to recombine at grain boundaries before they arrive the back contact, while free standing NWs array grown on conducting substrate avoids this shortcoming because the photo-generated charges can be transported to the substrate along individual nanowires which will enhance the photocurrent efficiency. The Effective use of Nanowires (NWs) require the ability to control and tailor their dimensions and morphology (height, diameter, spacing and planar density.

\section{ZnO NWs based Dye Sensitized Solar Cell (DSSC)}

Among the excitonic solar cells, the dye sensitized solar cell (DSSC), is considered a low cost solar cell. In a DSSC, dye molecules are used to sensitize wide-band gap semiconductors, such as $\mathrm{ZnO}$ and $\mathrm{TiO}_{2}$, which assist in separating electrons from photo-excited dye molecules. Gerishcer [4] and Memming [5] initiated in 1960 the sensitization of wide-band gap semiconductors by adsorbed monolayers of dye molecules. Thus 1-D wide-band gap semiconductor nanostructures were introduced to improve the charge collection (Figure 3a). The limitation of the DSSC is the poor absorption of low energy photons using available dyes. The redox electrolyte's instability is one of the current challenges, where it degrades over time under UV radiation and may leak if the cell is not perfectly sealed rendering the device inefficient for long term use [6].

\section{Zinc oxide (ZnO) Nanowires Based Quantum Dots Sensitized Solar Cell (QDSSC)}

$\mathrm{ZnO}$ based DSSCs have still lower conversion efficiencies, caused by :(1) instability of $\mathrm{ZnO}$ in acidic dyes by the formation of excessive $\mathrm{Zn}^{2+}$ /dye agglomerates; (2) decrease in the kinetics of electron- injection from dyes to $\mathrm{ZnO}$ caused by ultrafast electron injection that are opposed by molecular relaxation [7]. To overcome these drawbacks, semiconductor QDs have been used as photo-sensitizers to replace dyes [8]. Theoretically, the conversion efficiency of QDSSCs is expected to reach $44 \%$ that is considerably higher than that of DSSCs [9]. The co-sensitization of different sized QuatumDots [10] (Figure 4) will increase the adsorption coverage of solar spectrum and thus increase light harvesting efficiency, (Figure 3b). Many techniques are employed using smaller band-gap materials to expand the absorbance, which in turn increase the light harvesting and overall energy-conversion efficiency of the solar cell.

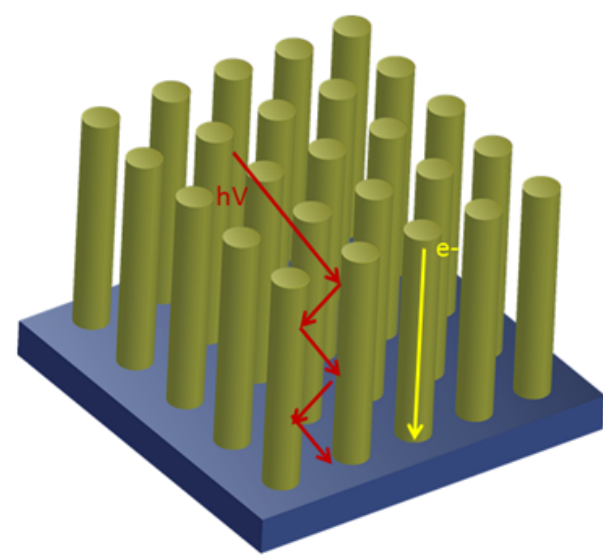

Figure 2: Schematic illustration for Nanowires in light trapping and electron transport to electrodes.

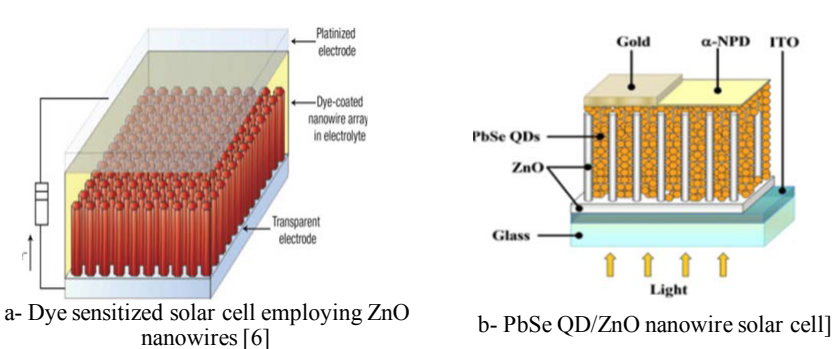

Figure 3: Schematic of ZnO Nanowires based a) DSSC , b) QDSSC

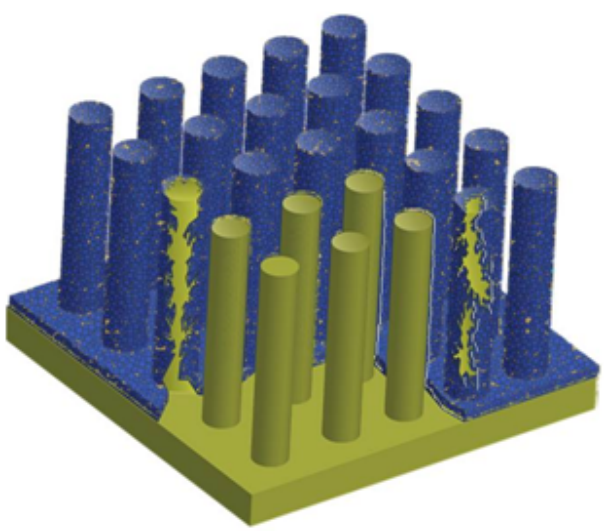

Figure 4: Quantum Dots Sensitized solar cells 
Citation: El-Zein B (2013) Self-Sufficient Energy Harvesting in Robots using Nanotechnology. Adv Robot Autom 2: 113. doi: 10.4172/21689695.1000113

\section{Other Applications Of Nanowires}

\section{Uv photodiodes \& optical switches}

Highly sensitive nanowire switches were demonstrated [11] by exploring the photo-conducting properties of individual semiconductor nanowires. The conductivity of the $\mathrm{ZnO}$ NWs is sensitive to ultraviolet light exposure. The increased light-induced conductivity allows reversibly switching the nanowires between "OFF" and "ON" states, an optical gating phenomenon analogous.

\section{Horizontal zno nanowires waveguides}

The wave guiding properties $[12,13]$ of single $\mathrm{ZnO}$ Nanowires have been investigated by coupling external light into the wire, it is noticed that the light is guided to both ends of the $\mathrm{ZnO} \mathrm{NW}$ from where it is emitted. While significant scattering is observed in the coupling region, no additional wave guiding losses along the $\mathrm{ZnO}$ Nanowires are observed (Figure 5,6).

\section{Zno nanowires lasers}

One of the most important and widely investigated applications of $\mathrm{ZnO}$ NWs is optically pumped ultraviolet lasing. Since the first report by Huang et al [14], many research groups have studied the mechanism of optically excited lasing in $\mathrm{ZnO}$ micro- and nanostructures including micropillars, NWs, and nanorods $[15,16]$. High-quality singlecrystalline $\mathrm{ZnO}$ NWs constitute ideal lasing nanocavities, which provide both a gain medium and a resonant cavity due to reflection at the planar end-facets.

\section{Nanotechnology-enabled piezoelectric mechanical-energy harvesting}

Vibration-based mechanical energy is abundant in the environment and more accessible than solar and thermal energy [17,18] Several methods have been established for the conversion of mechanical energy into electricity through the use of piezoelectric materials [19]. Piezoelectric materials have received enormous attention owing to the ability of these materials to convert mechanical energy into electricity directly.Lead zirconatetitanate(PZT), and $\mathrm{ZnO}$ nanowires have been demonstrated as the most materials used for mechanicalenergy harvesting [20,21] Piezoelectric NWs excel their bulk counterparts in terms of their enhanced piezoelectric effect, superior mechanical properties, and extreme sensitivity to vibrations of ultra-small magnitude[22,23].

\section{Thermoelectric energy harvesting}

An increased interest was given lately to thermoelectric (TE) materials for electric power generation through direct energy harvesting from natural heat source or heat waste. TE effects will be used to generate electricity, to measure temperature, and to cool or heat the devices. Such phenomena include See beck effect (conversion of temperature differences directly into electricity), Peltier effect (production of temperature difference by electric current), and Thomson effect (heating or cooling of a current-carrying conductor with a temperature gradient).An important property of the TE materials is that they are good electrical conductors but bad heat conductors. However, in an environment that the temperature is uniform, the pyroelectric effect has to be used to convert the heat into electricity [24] $\mathrm{ZnO}$ (Figure 7) with its pyroelectric and semiconducting properties creates a polarized electric field and charge separation along the nanowires based on timedependent change in temperature.

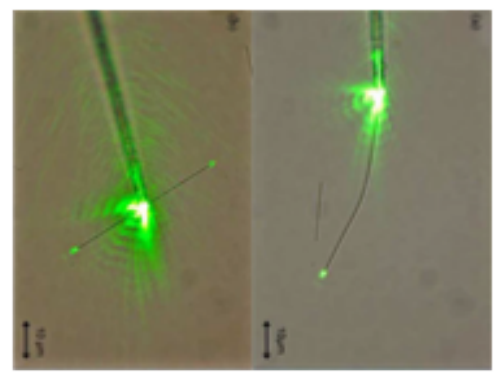

Figure 5: Coupling of green laser light from a silica-tapered fiber into a $\mathrm{ZnO}$ NW (a) from one end and (b) at the middle. Image source: Image reproduced fromo.

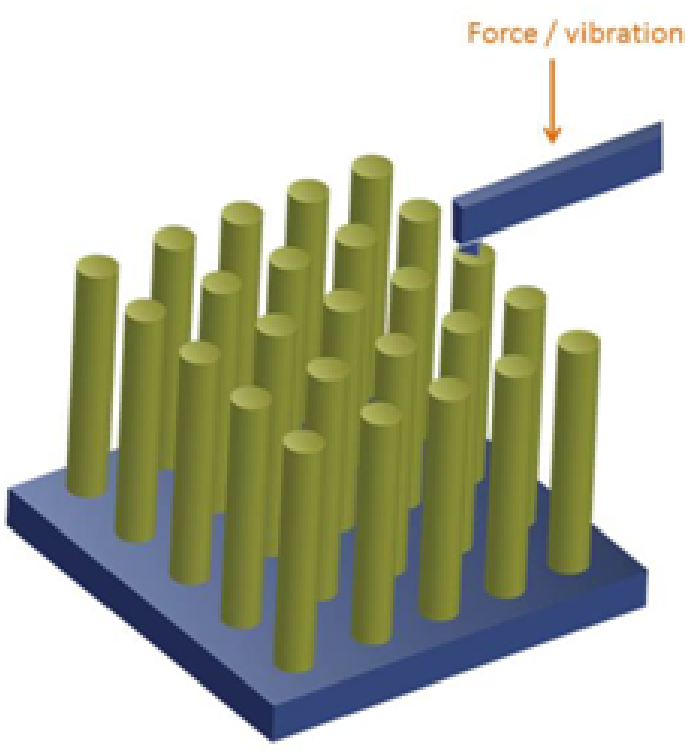

Figure 6: Nanowires arrays with piezoelectric property.

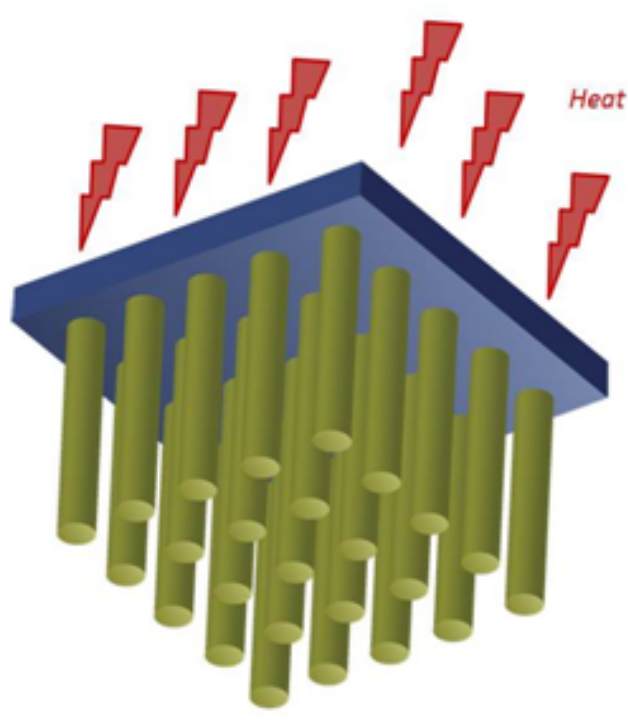

Figure 7: Thermoelectric ZnO NWs Arrays. 
Citation: El-Zein B (2013) Self-Sufficient Energy Harvesting in Robots using Nanotechnology. Adv Robot Autom 2: 113. doi: 10.4172/21689695.1000113

\section{Conclusion}

Electronic devices used in different sectors such as environment, industry, healthcare and telecommunications are empowered by rechargeable batteries. Using these batteries may result in many challenges in controlling environment pollution, extending the lifetime, recycling and replacing them, In order to effectively solve these challenges, researchers are putting lots of effort on the development of technology using nanostructures and nano-materials to harvest energy from the environment in which those electronics devices are used.

In this article we presented the importance of Nanotechnology in energy harvesting applied in nano-robots. It is expected that this subject create a new revolution world and miracles in the current science and technology.Future engineers, researchers and the scientists have opened the door for more research in the field of nano-robot to improve the device efficiency and improve the different types of energy harvesting devices using new nano-materials and nanostructures.

\section{References}

1. B.ElZein, E.Dogheche , Ch2, Lampert academic publishing ISBN: 978-3-65944003-8

2. Fan Z, Ruebusch DZ, Rathore AA, Kapadia R, Ergen E, et al. (2009) Challenges and Prospects of Nanopillar Based Solar Cells. Nano Research 2:829-843.

3. Ford AC, Ho JC, Chueh YL, Tseng YC, Fan Z et al.(2009) Diameter-Dependent Electron Mobility of In As Nanowires. Nano Letters 9: 360-365.

4. Gerischer H (1972) Electrochemical Techniques for the Study of Photosensitization. Photochem Photobiol 16: 243-260.

5. Memming R (1972) Photochemical and Electrochemical Processes of Excited Dyes at Semiconductor and Metal Electrodes. Photochem Photobiol 16: 325333

6. Michael Grätzel (2003) Dye-sensitized solar cells. Jou Photochemistry and Photobiology C: Photochemistry Reviews 4:145-153.

7. Qifeng Z, Christopher SD, Xiaoyuan Z, Guozhong C (2009) ZnO Nanostructures for Dye-Sensitized Solar Cells. Advanced Materials 21:4087-4108.
8. Chen J, Song JL, Sun XW, Deng WQ, Jiang CY, et al. (2009) An oleic acidcapped CdSe quantum-dot sensitized solar cell. Appl Phys Lett.

9. Feifei G,Yuan W,Jing Z,Dong S, Mingkui W, et al. (2008) A new heteroleptic ruthenium sensitizer enhances the absorptivity of mesoporous titania film for a high efficiency dye-sensitized solar cell.Chemical Communications 23: 26352637.

10. Chih HC, Shoou JC, Sheng PC, Meng JL, Cherng IC et al.(2010) Fabrication of a White-Light-Emitting Diode by Doping Gallium into ZnO Nanowire on a p-GaN Substrate. J Phys Chem C 114: 12422-12426.

11. H.Kind,H.Yan,B.Messer,M.Law,P.Yang,Adv.Mater 2002,14, No2

12. Robert H, Heinz K (2006) Guided modes in ZnO nanorods. Appl Phys Lett.

13. T. Voss, Advances in Solid State Physics, vol. 48. Berlin, , p. 57, 2009.

14. Michael HH, Samuel M, Henning F, Haoquan Y, Yiying W, et al. (2001) RoomTemperature Ultraviolet Nanowire Nanolasers. Science 292:1897-1899

15. J. Fallert et al.,Opt. Express, vol. 16, no. 2, pp. 1125-1131, Jan. 2008.

16. M. A. Zimmler, J. Bao, F. Capasso, S. M"uller, and C. Ronning, Appl. Phys. Lett., vol. 93, no. 5, pp. 051101-1-051101-3, 2008

17. J. A. Paradiso, T. Starner, IEEE Pervas. Comput. 2005, 4, 18- 27

18. Cian ÓM,Terence OD,Rafael VMC,James R,Brendan OF (2008) Energy scavenging for long-term deployable wireless sensor networks. Talanta 75:613623.

19. Bouendeu E, Greiner A, Smith PJ, Korvink JG (2011) Design Synthesis of Electromagnetic Vibration-Driven Energy Generators Using a Variational Formulation. Microelectromechanical Systems Jou 20:466-475.

20. H. Chen, C. Jia, C. Zhang, Z. Wang, C. Liu in 2007 IEEE International Symposium on Circuits and Systems, Vols. $1-11,2007$, pp. $557-560$.

21. Youfan Hu, Yan Zhang, Chen Xu, Guang Zhu, Zhong LW (2010) High-Output Nanogenerator by Rational Unipolar Assembly of Conical Nanowires and Its Application for Driving a Small Liquid Crystal Display. Nano Lett 10:5025-5031.

22. Xudong Wang (2012) Piezoelectric nanogenerators Harvesting ambient mechanical energy at the nanometer scale. Nano Energy 1:13-24.

23. Zhong Lin Wang (2009) $\mathrm{ZnO}$ nanowire and nanobelt platform for nanotechnology.Materials Science and Engineering: R: Reports 64:33-71.

24. Ya Yang,Wenxi Guo,Ken CP,Guang Z,Yusheng Z, et al.(2012) Pyroelectric Nanogenerators for Harvesting Thermoelectric Energy.Nano Lett.12: 2833 2838. 